\title{
Application of spray techniques for new photocatalytic gradient coatings on plastics
}

\author{
H. Schmidt, M. Naumann, T.S. Müller*, M. Akarsu \\ Leibniz-Institut für Neue Materialien (INM), Chemistry and Technology of Materials-Catalysis, Saarbrücken, Germany
}

\begin{abstract}
Transparent coating systems applicable on plastics surfaces by a spray technique are presented. The coatings are based on highly photocatalytically active nanoscaled titania powders, surface modified with silanes containing organic or fluoro organic side chains. The modification allows for the introduction of the particles in organic inorganic hybrid NANOMER ${ }^{\circledR}$ coating systems. In the wet film due to the evaporation of the solvents a decompatibilisation of the coated particles to the matrix results in a self organising gradient layer formation with an up concentration of the active particles at the interface layer between coating and air. After activation by irradiation with artificial or natural UV light, highly active transparent photocatalytic coatings for a great variety of materials are obtained.
\end{abstract}

Keywords: Photocatalysis; Polymers; Gradient coatings; Titanium dioxide; Nanoparticles

\section{Introduction}

In 1972 Fujishima and Honda [1] described the effects of the irradiation of titania by UV-light. The development of a high oxidative potential of illuminated $\mathrm{TiO}_{2}$, especially the anatase modification, was described as the photocatalytic effect. The effect is caused by the formation of an electronhole pair in the semiconducting material if the photon energy exceeds the band gap, so for anatase wavelengths less than $388 \mathrm{~nm}$ are needed. Due to the diffusion of hole and electron to the surface, an oxidation potential of $3.2 \mathrm{eV}$ is obtained. On the surface aggressive species such as hydroxyl- or hydroperoxidyl-radicals are generated from moisture and atmospheric oxygen. These species easily attack and destroy organic substances in their vicinity. The effect is described in greater detail elsewhere [2,3].

This photocatalytic principle found at first mainly applications concerning the decomposition of organic contaminants and was discussed as an advanced oxidation technique for the treatment of water and air [4-6]. Later on

\footnotetext{
* Corresponding author.

E-mail address: tmueller@inm-gmbh.de (T.S. Müller).
}

it was discovered that illuminated titania also exhibited superhydrophilic properties, which were exploited for various applications with an emphasis on the self-cleaning, anti-fogging and antimicrobial effects [7].

A direct application of photocatalytically titania on organic materials like plastics, e.g. PC or PET, leads to a degradation of the substrates, resulting in the delamination of the coatings. To inhibit substrate degradation, it is necessary to provide a barrier layer between the photocatalyst and the polymeric substrate. Double- or multilayer systems have been developed, which do not allow a singlestep application, thus being time consuming and causing additional costs compared to single layer coatings.

To circumvent this problem at INM a single-step coating system was developed, which does not depend on the separate application of a barrier layer. This approach is based on experimental results achieved in the nineties of the last century at INM when the self-organising gradient formation in sol-gel materials was discovered. Suitably surface modified nano particles in organic-inorganic hybrid coating matrices will enrich at the interface between coating and air during the drying of the applied wet film, leading to the formation of a gradient in the particle concentration [8]. This principle was adapted to titania particles allowing for 
the formation of photocatalytically active gradient coatings [9]. The organic-inorganic matrix systems can be tailored to special requirements and are well known at INM (e.g. NANOMER ${ }^{\circledR}$-coatings). Besides their functionality they have the advantage of easy application methods like spray coating, dip coating and flow coating.

The work presented refers to first results of the adaptation of these coating systems on plastic materials applied by spray coating.

\section{Experimental}

\subsection{Synthesis of anatase nanoparticles by a reflux process}

$162.45 \mathrm{~g}$ 1-pentanol were mixed at room temperature with $107.51 \mathrm{~g}$ titanium tetraisopropylate in a roundbottomed flask under heavy stirring. After 2 min mixing time $7.458 \mathrm{~g}$ of a $37 \mathrm{wt} \% \%$ hydrochloric acid were added dropwise while continuing the agitation. Ten minutes later $7.973 \mathrm{~g}$ water were added in the same way. During the addition of the water, the solution turned opaque. When amorphous particles or a white precipitate was observed, the continuation of the process did not yield redispersible nanoparticles. The solution was stirred for another $20 \mathrm{~min}$ and the flask afterwards was transferred into a heating bath preheated to $135{ }^{\circ} \mathrm{C}$. The mixture was held at boiling temperature under reflux conditions for $16 \mathrm{~h}$. The reaction mixture was centrifuged (Megafuge $2.0 \mathrm{R}$ by Heraeus, $500 \mathrm{ml} \mathrm{PP-centrifugation} \mathrm{vessels} \mathrm{at} 4000 \mathrm{rpm}$ for $30 \mathrm{~min}$ ), the liquid phase was disposed. The solid paste was transferred into a round-bottomed flask and dried using a rotary evaporator at $40{ }^{\circ} \mathrm{C}$ bath temperature and reduced pressure. Further drying could be executed in an evacuated drying chamber over night at $40{ }^{\circ} \mathrm{C}$. About $14 \mathrm{~g}$ of a slightly yellowish powder was obtained. The powder was completely redispersible in toluene up to approx. 20 wt.\% of nano titania with a particle size of 4-6 nm (Fig. 1).

\subsection{Surface modification of the anatase nanoparticles obtained by the reflux process}

$1.007 \mathrm{~g}$ of the dry titania nanoparticles obtained from the reflux process were dispersed in $9.075 \mathrm{~g}$ of dry toluene by ultrasonic agitation for $15 \mathrm{~min}$. This sol was centrifuged at $8000 \mathrm{rpm}$ in $45 \mathrm{ml}$ PP centrifuge vessels (Dow-Corning) using a Hermle Z323K centrifuge in order to remove agglomerates. The solids were discarded and the sol was transferred in a round bottom flask, mixed with $0.234 \mathrm{~g}$ 3,3,4,4,5,5,6,6,7,7,8,8,8-tridecafluorooctyl-1,1,1-triethoxysilane (FTS) and stirred in the dark for $16 \mathrm{~h}$. Afterwards the solvents were evaporated using a rotary evaporator at $40^{\circ} \mathrm{C}$ bath temperature and reduced pressure to obtain a dry powder. Further drying could be executed in an evacuated drying chamber over night at $40{ }^{\circ} \mathrm{C}$.

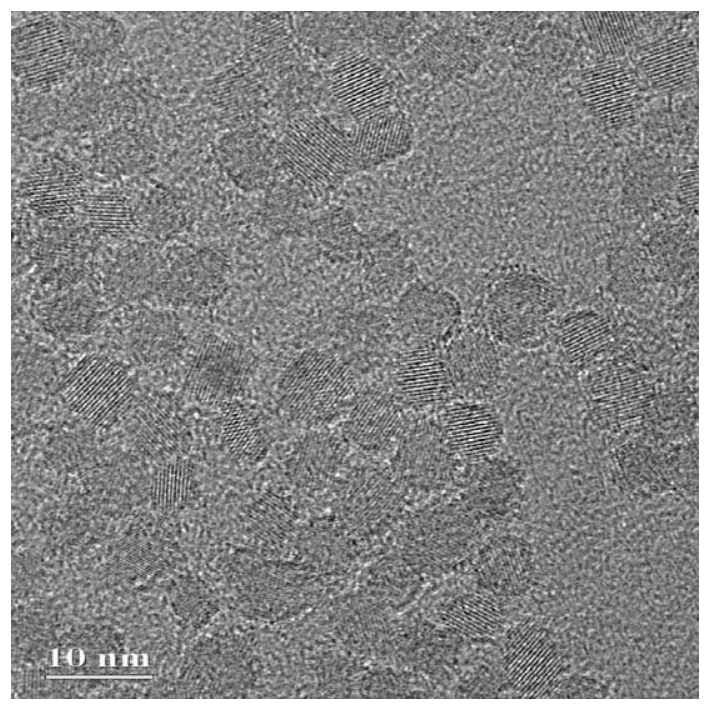

Fig. 1. TEM microphotograph of anatase particles.

\subsection{Preparation of the coating materials}

\subsubsection{Binder}

$36.6 \mathrm{~g}$ methyltriethoxysilane (MTEOS), $11.59 \mathrm{~g}$ tetraethoxysilane (TEOS) and 20.4 g Levasil 300/30 (an aqueous silica sol, Bayer AG) were mixed and stirred vigorously. $0.43 \mathrm{~g}$ of $32 \mathrm{wt} . \% \mathrm{HCl}$ were quickly added in one step. The reaction is exothermous and the mixture was stirred for 30 min until the sol was cooled down to room temperature. The resulting sol was diluted with $72 \mathrm{~g}$ isopropanol (other diluents have been used as well, as described in the "results" part). The diluted sol was filtrated using a 0.8 $\mu \mathrm{m}$ filtration membrane. This "binder" sol was stable in a refrigerator for at least 2 months.

\subsubsection{Composite coating material}

For the preparation of the photocatalytic coatings $150 \mathrm{mg}$ FTS modified $\mathrm{TiO}_{2}$-particles were suspended in $12 \mathrm{~g}$ of dry MEK (methylethylketone) under sonification. This mixture was added to $50 \mathrm{~g}$ of the binder sol and treated in an ultrasonic bath until a completely transparent suspension was obtained.

\subsubsection{Coating procedure}

The application of the coatings on PVC was done by spray coating and also by dip coating, spin coating or flow coating. For optimal adhesion a pre-treatment of the plastic substrates with primers (e.g. a prehydrolized solution of aminopropyltrimethoxysilane in 1-butanol [10]), $\mathrm{Ar} / \mathrm{O}_{2}-\mathrm{RF}-$ plasma or surface flame impingement (SurASil 600, SurA Chemicals, Jena, Germany) was used. After the application, the wet film was allowed to pre-dry at room temperature before the coated substrates were transferred into an oven for at least $1 \mathrm{~h}$ at $110{ }^{\circ} \mathrm{C}$. The optimal thickness of these coatings was recognised to be around $1-2 \mu \mathrm{m}$, the viscosity of the coating sols could be adjusted by the addition of diluents like isopropanol. The photocatalytic coatings could 


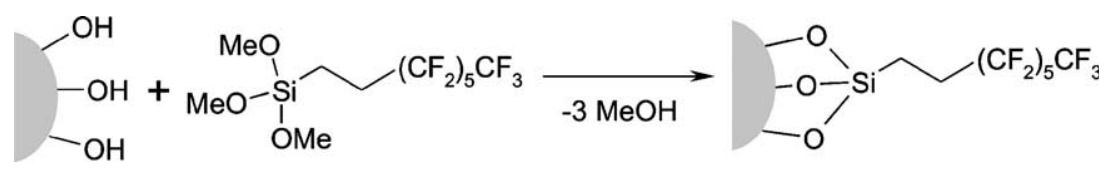

Fig. 2. Scheme of the surface modification of the nanoparticulate titania.

easily be activated by irradiation with UV-light (e.g. from a low pressure Hg-lamp) or direct sunlight.

\section{Results and discussion}

In order to obtain transparent coatings, nanoparticulate titania had to be used as the active material. Given the high refractive index of $\mathrm{TiO}_{2}$ of 2.52, the particle size of the anatase had to be significantly below $20 \mathrm{~nm}$. Only particles this small do not scatter light, which would result in white coatings. In Fig. 1, the particles obtained according to Section 2.1 are shown. These particles were surface modified with fluororganic silanes and incorporated in a binder system.

The anatase nanoparticles were obtained by a reflux process as described above. The preparation route also allowed for an easy doping of the particles by transition metal ions, providing photocatalysts sensitive to visible light [11]. The particles obtained had primary particle sizes of 4$6 \mathrm{~nm}$, as also can be seen in the TEM micrograph in Fig. 1. They are easily redispersible in toluene to a transparent colloidal solution, what can be attributed to remaining alkoxy groups covering the surface of the particles and leading to a hydrophobic surface. Chemical analysis revealed that the particles contained about $18-19 \%$ organic carbon.

In order to obtain the desired self-organising coatings and to optimise the gradient development process, the polarity of the particles had to be further decreased by a surface modification with a fluoro-organic silane $(3,3,4,4,5,5,6$, 6,7,7,8,8,8-tridecafluorooctyl-1,1,1-triethoxysilane [FTS]) as schematically presented in Fig. 2 .

For the investigation of the self-organising coatings the modified titania nanoparticles were redispersed in methylethylketone (MEK) using an ultrasonic bath. These sols were mixed with inorganic-organic matrix sols (binders). In the past, these binders and the so-called NANOMER ${ }^{\circledR}$ coating systems have been successfully used for many applications which are described elsewhere in greater detail [12]. NANOMER ${ }^{\circledR}$ coatings are obtained by hydrolysing various functionalised and nonfunctionalised silanes in the presence of inorganic functional nanoparticles, e.g. silica or zirconia.

Freshly prepared coating sols containing FTS-modified titania nanoparticles look completely homogenous and no phase separation is observed. After the application of the coating sol, the different solvents (e.g. MEK, ethanol and water) evaporate with different rates from the wet film. This results in a gradual change of the balance between polar and nonpolar compounds in the film, finally leading to a thermodynamically driven decompatibilisation between the modified particle surface and the matrix system. Since the photocatalytic nanoparticles have a size of less than $10 \mathrm{~nm}$, they can easily diffuse towards the interface between coating and air, which is schematically shown in Fig. 3.

The process of the gradient formation raised the question whether the application of the coating by spraying would change the balance between polar and nonpolar solvents during the spray process by solvent evaporation from the droplets. First tests using a material as described under Section 2.3, however, delivered a coating which could be successfully activated and showed photocatalytic properties. The quality of the coating, reflected in terms such as optical homogeneity of the coating and photocatalytic activity, was significantly improved by lowering the viscosity of the coating material, indicating that by keeping the viscosity lower, the up-concentration of the nanoparticles at the film surface is improved due to a higher diffusion rate. By diluting the binder with triple the amount of isopropanol compared to Section 2.3.1 it also proved positive if $10 \%$ of the volume of the isopropanol was exchanged by the same
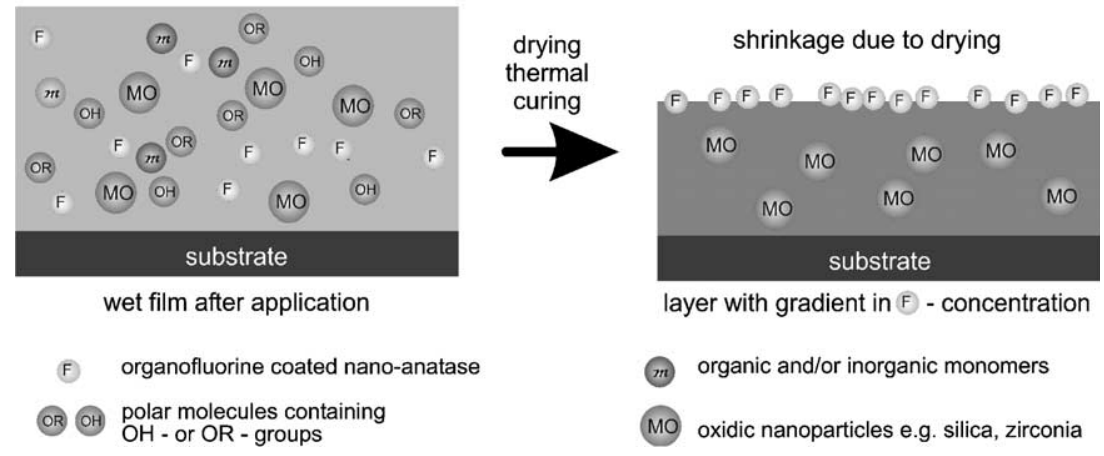

Fig. 3. Proposed mechanism of the gradient formation. 

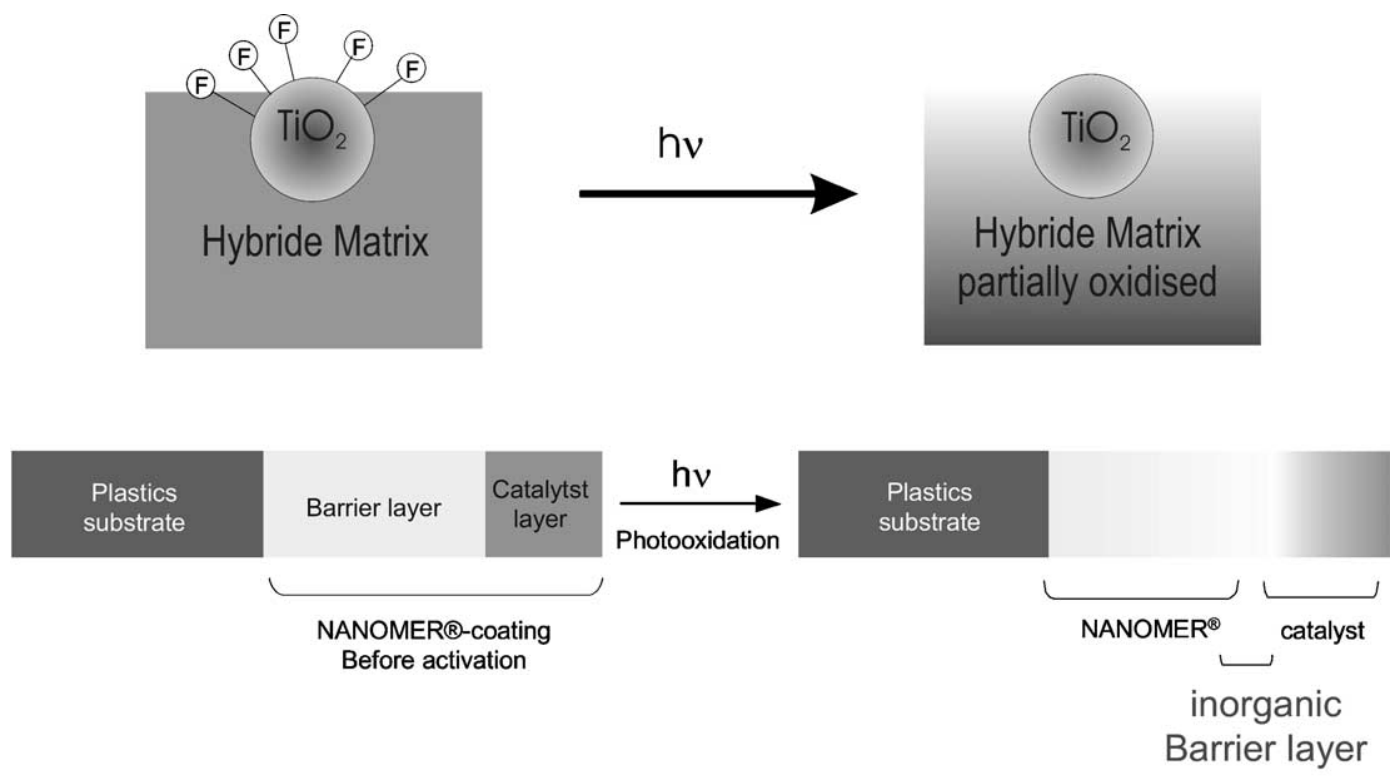

Fig. 4. Scheme of the photoactivation of the gradient coating system

volume of isopropoxyethanol (IPE), a solvent with a higher boiling point. When higher amounts of low volatile solvents were used, sticky coatings were obtained even after curing times of longer than $24 \mathrm{~h}$.

The adhesion of the coatings was determined by a combination of a cross-cut cc (DIN EN ISO 2409) together with a tape test tt (DIN 58196-6). Adhesion on PVC (white, about $30 \%$ of plasticizers, for architectural use) was optimal $(\mathrm{cc}=0 / \mathrm{tt}=0)$ after cleaning with isopropanol followed by plasma treatment.

During the activation of the photocatalytic coating, which can be easily achieved by irradiation with UV-light, either artificial (e.g. using a Hereaus Suntester for 1-2 h) or sunlight (about 3-5 days at a clear sky), the organic side chains of the surface modification of the titania particles are oxidised revealing the active form of the particles. During this process the contact angle against water is reduced from values of $90-100^{\circ}$ to values below $10^{\circ}$. In this case water drops will spread to thin water films, the surface becomes superhydrophilic. Furthermore the organic content of the matrix system in the vicinity of the photocatalytically active titania nanoparticles will be oxidized as well. This leads to an intrinsic barrier layer $\left(\mathrm{SiO}_{2}\right)$ in the coating which is protecting sensitive polymeric substrates from photocatalytic oxidation which is schematically shown in Fig. 4. During an observation period of several months of outdoor exposure, no decay of the layer system and the organic substrates was observed.

The gradient formation was confirmed by analytical methods. SIMS analysis of coated PC sheets showed a significant decrease in the Ti signal intensity in about 300 $\mathrm{nm}$ depth of the coating. Furthermore, the oxidation of the organic material in the vicinity of the titania nanoparticles and the formation of an inorganic barrier layer is proven by the decreasing carbon content in the same area after irradiation in a HERAEUS Suntester for several hours.
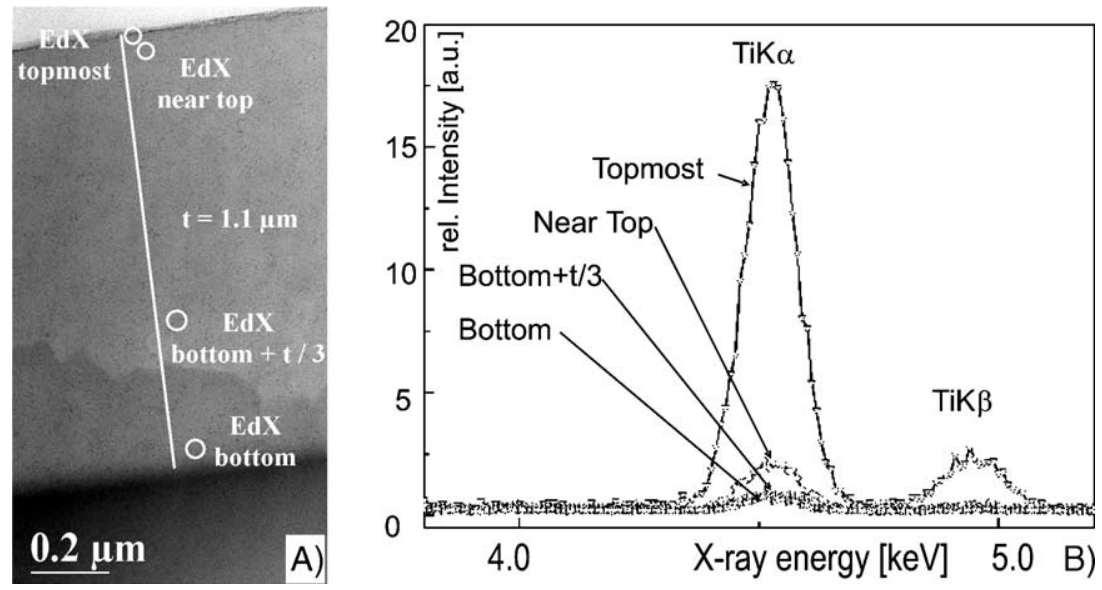

Fig. 5. TEM micrograph of a gradient layer having a thickness of $1.1 \mu \mathrm{m}$ with the positions of 4 EDX measurements (left); EDX experiments showing the titanium $K_{\alpha}$ and $K_{\beta}$ signals measured at the 4 positions (right). 


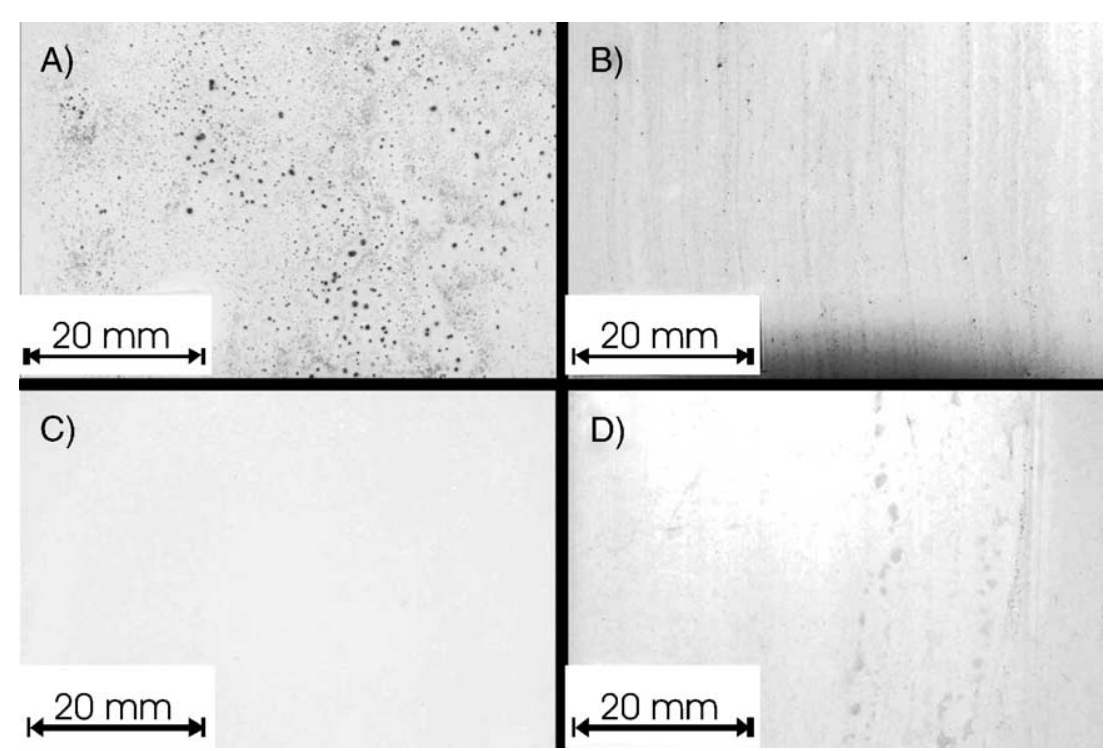

Fig. 6. Samples of white PVC: Left column (a, c) photocatalytically coated, right column (b, d) not coated. a, b: after soiling with street dirt using a brush, c, d: after a 20 -min rain shower.

The enrichment of the titania particles at the surface of the coating could also be shown by TEM and EDX-analysis (Fig. 5). For a coating on glass, the steep gradient structure in the coating has been made visible by the TEM micrograph on the left of the figure. The top light gray triangle shows the resin for the fixation of the specimen, the black part at the bottom shows the glass substrate. Close to the surface of the coating an enrichment of the titania particles at the interface between the coating and air can be seen (particles appear as dark spots due to the crystalline nature), whereas almost no particles were found in the depth of the coating. To support the visual impression, four EDX measurements were executed (indicated by the white circles). The right part of Fig. 5 shows the spectra of these four EDX experiments. Only in the topmost position a significant signal for Ti could be found, whereas the deeper coating is almost free from titanium. These results will be discussed in greater detail elsewhere [13].

These newly developed gradient materials are showing self-cleaning and anti-fogging behaviour as well as a degradation of organic contaminants as it is already known of common photocatalytically active surfaces. The selfcleaning effect is demonstrated by a spray coated white PVC substrate in comparison to an uncoated sample. In Fig. 6 the left column (a, c) shows the coated photocatalytically active material, the right column $(b, d)$ shows the uncoated material. For an experiment the samples were soiled with a brush by applying a street dirt mixture at the different surfaces. Afterwards the soiled samples were allowed to dry up. The resulting surfaces are shown in the top row $(\mathrm{a}, \mathrm{b})$, it can be seen that the soiling behavior differs between the different surfaces. The samples were exposed outdoors and were photographed again after a rain shower of about $20 \mathrm{~min}$. In case of the coated sample the rain was able to wash off the dirt completely (c), while in case of the uncoated reference sample stains still could be observed (d).

\section{Conclusions}

The presented technique for the preparation of photocatalytic gradient coatings based on nanoparticulate titania opens up a new way for the manufacture of highly photocatalytically active surfaces on plastic materials. The small particle size of the titania used together with the presented surface modification of the nanoparticles enables the introduction into coating systems in a very simple way to form transparent photocatalytic coatings on various substrates.

\section{Acknowledgement}

The authors wish to thank the Government of the Federal Republic of Germany, the State of Saarland, and various industrial companies for their financial support and R. Karos and Dipl. Phys. K. Moh for their experimental help and their fruitful discussions.

\section{References}

[1] K. Honda, A. Fujishima, Nature 238 (1972) 37.

[2] A.L. Linsebigler, G. Lu, J.T. Yates, Chem. Rev. 95 (1995) 735.

[3] M.A. Fox, M.T. Dulay, Chem. Rev. 93 (1993) 341.

[4] M. Levandowski, D.F. Ollis, in: V. Ramamurthy, K.S. Schanze (Eds.), Semiconductor Photochemistry and Photophysics, Marcel Dekker Inc, New York, 2003, p. 249.

[5] P. Pichat, in: M.A. Tarr (Ed.), Chemical Degradation Methods for Wastes and Pollutants, Marcel Dekker Inc, New York, 2003, p. 77. 
[6] D.F. Ollis, H. Al-Ekabi, Photocatalytic Purification and Treatment of Water and Air, Elsevier, Amsterdam, 1993.

[7] A. Fujishima, K. Hashimoto, T. Watanabe, $\mathrm{TiO}_{2}$ Photocatalysis: Fundamentals and Applications, BKC, Tokyo, 1999.

[8] H.K. Schmidt, Glass Sci. Technol. (Glastech. Ber.) 68 (C1) (1995) 21.

[9] M. Akarsu, E. Arpac, H. Schmidt, DE 10235803 A1, 5.8. (2002).

[10] U. Nolte, "Untersuchung der Haftmechanismen thermisch härtbarer Sol-Gel-Nanokomposit-Materialien auf Kunststoffen bei Verwendung von Aminosilanen als Primer", doctoral thesis, 2001, Naturwissenschaftlich-Technische Fakultät III, Chemie, Pharmazie und Werkstoffwissenschaften der Universität des Saarlandes.

[11] H. Schmidt, M. Akarsu, Th.S. Müller, K. Moh, G. Schäfer, D.J. Strauss, M. Naumann, Res. Chem. Intermed. 31 (4 6) (2005) 535.
[12] (a) S. Goedicke, "Entwicklung und Charakterisierung eines hochtemperaturbeständigen Bindemittels für Dämmstoffe aus Glaswolle“, doctoral thesis, 2001, Naturwissenschaftlich-Technische Fakultät III, Chemie, Pharmazie und Werkstoffwissenschaften der Universität des Saarlandes;

(b) M. Mennig, G. Jonschker, H. Schmidt, Glas-Ing. 3 (1994) 54; (c) M. Mennig, G. Jonschker, H. Schmidt, Sol-Gel Opt. II 1758 (1992) 125 ;

(d) Patents: DE 4217432, DE 19647368, DE 19647369, DE 19915377, EP 642475, EP 729442.

[13] Th. Krajewski, M. Naumann, K. Moh, T.S. Müller, M. Akarsu, H. Schmidt (in preparation). 